\title{
EL VACIAMIENTO DE LOS DERECHOS HUMANOS EN LA ESTRATEGIA DE GLOBALIZACIÓN (LA PERSPECTIVA DE UNA ALTERNATIVA) ${ }^{1}$
}

\author{
THE DISREGARD OF HUMAN RIGHTS IN THE GLOBALIZATION STRATEGY (AN \\ ALTERNATIVE SOCIETY PERSPECTIVE)
}

\section{Franz Hinkelammert ${ }^{2}$}

\begin{abstract}
Resumen
El artículo discute las bases de los derechos humanos en el mundo de hoy, y presenta la tesis de que ha habido un cambio profundo en la visión de los derechos humanos de parte de los poderes económicos y políticos a partir de la llamada estrategia de globalización, que de hecho es una estrategia del mercado total.

También se analizan los rasgos constitutivos del Estado social posterior a la segunda guerra mundial y cómo sus elementos claves hoy son percibidos por los sectores dominantes como políticas de izquierda. Lo anterior unido, especialmente en América Latina, a la sustitución de las democracias controladas por los Estados de seguridad a partir del golpe militar chileno en 1973 y hasta finales del siglo XX. Esto comprueba que un capitalismo neoliberal no se puede sostener a la larga si no es como un sistema de mercado total sostenido por ideologías de Seguridad Nacional y sus correspondientes ejércitos.
\end{abstract}

Doi: http://dx.doi.org/10.15359/eys.21-49.4

Recibido el 27 de abril del 2016. Reenvíos el 23 de mayo del 2016. Aprobado el 24 de mayo del 2016. Publicado el 24 de junio del 2016

${ }^{1}$ Conferencia magistral: "El vaciamiento de los derechos humanos en la estrategia de globalización", en el Congreso de la Asociación Latinoamericana de Sociología (ALAS). Costa Rica, diciembre de 2015. Economía \& Sociedad ha realizado algunos cambios básicos de edición sobre el texto original. economiaysociedad@una.cr

${ }^{2}$ Economista y teólogo alemán. Doctor en Economía por la Universidad Libre de Berlín. Cofundador del Departamento Ecuménico de Investigaciones (DEI), en San José, Costa Rica. Exponente de la teología de la liberación y de la crítica teológica al capitalismo. Actualmente es consultor independiente. 
Los nuevos proyectos de sociedad alternativa que han surgido en América Latina, responden precisamente al totalitarismo de mercado que fomenta el neoliberalismo.

Palabras claves: derechos humanos; Neoliberalismo; mercado total; Estado Social; sociedad alternativa

\begin{abstract}
Human rights foundations in today's world are discussed. The author presents the thesis that there has been a profound change on how human rights are viewed by the economic and political powers due to the so-called globalization strategy, which, in fact, is an overall market strategy.

The author also analyzes the constituent features of the Social State after World War II and how dominant sectors currently perceive its key elements as left-wing politics. The former is added to the replacement of democracies controlled by Security States, particularly in Latin America, from the Chilean military coup in 1973 to the end of the 20th century. This proves that neo-liberal capitalism cannot be sustained in the long run if it is not treated as a total market system held by ideologies of National Security and their respective armies.
\end{abstract}

The new alternative society projects that have emerged in Latin America exactly respond to the market totalitarianism promoted by neo-liberalism.

Keywords: human rights; neoliberalism; total market; Social State; alternative society

Creo, que es necesario hoy, volver a discutir las propias bases de los derechos humanos en el mundo. Ha habido un cambio profundo de la visión de los derechos humanos de parte de los poderes tanto económicos como políticos al iniciarse la tal llamada estrategia de la globalización bajo el gobierno de Ronald Reagan en EE. UU. En Latinoamérica no hubo inicialmente mucha respuesta, quizás por el hecho de que las dictaduras de Seguridad Nacional impuestas con el apoyo de EE. UU., hacían imposible una respuesta significante. Eso ciertamente cambió después y especialmente desde fines del siglo pasado, porque aparece un movimiento antiglobalización. También surgen ahora movimientos amplios de reformulación de la política y con ello de la relación con los derechos humanos, que inclusive tomaron el poder político. Los más fuertes aparecieron en Venezuela, Bolivia y Ecuador.

Pero a la vez hay una respuesta, en la cual se vota con los pies. Los movimientos de refugiados, sin casi ninguna organización, fueron en aumento. Desde mucho tiempo también entre América 2 
Latina y EE. UU., pero cada vez más desde el cercano oriente y África hacia Europa, empujados todavía más por el surgimiento de organizaciones terroristas y guerras cada vez más amplias. Los movimientos de los indignados aparecieron en el norte de África y encontraron rápidamente repercusión muy amplia en España y en varios países de América Latina.

Pero lo que hoy más se nota, es el movimiento de refugiados de los países del medio oriente y de África del norte. Ciertamente, las guerras lo empujan. Pero de ninguna manera son la única razón. En el fondo de todo están las consecuencias de la estrategia de globalización, que arrasa con los países que no logran integrarse en el movimiento general. Las poblaciones pierden el arraigo en su propia tierra, no ven ningún futuro esperanzado, por lo que están dispuestos a lanzarse, aunque corran el riesgo de muerte. Son tantos los que se mueven, que la pura acción violenta frente a ellos parece imposible, de tal manera que tendría que ser tanta la violencia en contra de estos refugiados, que ninguna opinión pública se puede formar para apoyarla. Además hay una gran sorpresa que los Estados afectados tienen que enfrentar: las poblaciones europeas apoyan muchas veces en sus mayorías una recepción humanitaria de estos refugiados. Toda la brutalización de las relaciones humanas, que fue cultivada por los estrategas de la globalización, no ha logrado brutalizar suficientemente estas mayorías, y la mayoría de los gobiernos se ven en la necesidad de respetar eso.

\section{El período después de la II Guerra Mundial y la reconstrucción de Europa occidental}

Tenemos que preguntarnos, por tanto, cuál fue la razón para implementar esta estrategia de globalización, que de hecho es una simple estrategia del mercado total, por tanto hasta se podría hablar de un totalitarismo del mercado.

La estrategia de globalización se desarrolló en los años 70 del siglo pasado. Asimismo, empezó con el golpe militar chileno y su dictadura de Seguridad Nacional en 1973. A este siguió Margaret Thatcher como primera ministra de Inglaterra a partir de 1979 y la llegada a la presidencia de EE. UU. de Reagan en 1980. Ellos terminaron con el período anterior, que empezó con el fin de la II Guerra Mundial.

Tenemos que enfocar precisamente este período de reconstrucción de Europa Occidental y sus extensiones mundiales para poder evaluar lo que es esta estrategia de globalización. Es interesante hacer eso, porque los responsables de la estrategia de globalización más bien han puesto un gran manto de silencio sobre lo que ha sido. Por eso, nuestros medios de comunicación no nos informan casi nada.

Se trata primero del período de la misma reconstrucción de Europa después de la catástrofe de la II Guerra Mundial. Pero esta reconstrucción se efectúa en condiciones políticas muy especiales. EE. UU. se enfrenta en Europa con la Unión Soviética, que era uno de los Aliados que 
habían ganado la guerra. Pero ahora EE. UU. la consideró un enemigo peligroso, sobre todo por razones ideológicas. Era un país socialista, que trató de llevar a su población a una situación de satisfacción de necesidades en un grado alto y suficiente para todos. Los partidos comunistas en Europa occidental eran poderes muy significativos, sobre todo en Francia e Italia. EE. UU. por eso no necesitaba cualquier reconstrucción de los países de Europa Occidental, por lo tanto, tenía que ser una reconstrucción que mostrara hacia afuera un "capitalismo con rostro humano". Para un mundo capitalista se trataba de una meta inaudita. Pero el enfrentamiento con los esfuerzos del socialismo de la Unión Soviética era inevitable.

Solamente eso explica la disposición del mundo capitalista de lanzarse a construir una sociedad de pretensión humanitaria. Efectivamente se hizo eso en un grado significante. Seguía siendo una sociedad capitalista, pero de un capitalismo sumamente relativizado por un notable cumplimiento con los derechos humanos y la constitución sistemática de un Estado social y con una planificación económica eficaz, de apoyo a las metas generales del Estado social, pero entendiendo esta planificación como una de tipo indicativo. Era pensada desde la teoría económica de Keynes. Elementos claves de este Estado social son:

1. La anulación prácticamente general de todas las deudas de estos países. En parte las deudas son condonadas, en parte da una moratoria de las deudas sin pagar intereses por este período de reconstrucción.

2. Nuevos créditos del llamado plan Marshall, en parte sin devolución y en otra parte con tasas de interés muy bajas.

Este plan Marshall tuvo una gran importancia para la reconstrucción, exclusivamente por el hecho de que toda deuda anterior había sido anulada. En caso contrario el dinero del plan Marshall habría servido exclusivamente para financiar el pago de la deuda anterior sin ningún apoyo para la reconstrucción. Muchas veces se olvida mencionar eso como recientemente en el caso del tal llamado apoyo financiero para Grecia. En Grecia se trataba de un apoyo para las bancas extranjeras frente a las cuales Grecia estaba endeudada. Pero nuestro lenguaje hipócrita habla de ayuda para Grecia.

3. Impuestos especialmente altos para ingresos altos y para el capital, por eso de fuerte carácter progresivo, igualmente impuestos altos a las herencias y sobre las propiedades. El impuesto de renta de los ingresos más altos era en Alemania de 65\%.

4. La constitución del Estado social en cuanto a un sistema público de salud, un sistema público de educación, un sistema público de seguro de desempleo y de jubilación, una política de fomento de la construcción de viviendas populares. Se conserva el carácter público del correo, del ferrocarril, del agua, de buena parte de la electricidad.

4 
5. La creación de la Unión Europea de Pagos, que tenía el propósito de evitar la posibilidad de la formación de una deuda externa a partir de desequilibrios crónicos en la balanza de pagos. La convicción era que los superávits son tan dañinos como los déficits. Ambos, los países con superávit y los países con déficit son responsables en común, lo que inclusive implicaba la posibilidad de obligar a financiar los déficits por superávits sin posibilidad de cobrar intereses.

6. Se impuso a la vez el principio de asegurar un aumento regular de todos los ingresos con el ritmo de las tasas de crecimiento de la economía, para evitar una concentración acumulativa de los ingresos altos. Se trataba de evitar aumentos excesivos de la concentración de ingresos.

7. Fomento sistemático de organizaciones populares, sobre todo en el caso de los sindicatos.

8. Fomento de medios de comunicación -especialmente radio y televisión- de propiedad pública, que fueron organizados de manera autónoma y con la función de asegurar informaciones objetivas frente al riesgo de la distorsión de las informaciones por los medios privados. El modelo ha sido la BBC inglesa. Ha sido llamativo la Radiotelevisione Italiana RAI, que tenía uno de los pocos canales de televisión en oposición a Berlusconi y la casi totalidad de los otros canales en la mano del propio Berlusconi. La ley protegía la autonomía de los medios públicos, aunque eso molestaba a Berlusconi. En Alemania estaba prohibida hasta los años 80 la radio y la televisión privada.

Se trataba de un proyecto estratégico que tuvo un éxito extraordinario. Empezó con Europa, pero también marcó la política del desarrollo del mundo occidental. La política de desarrollo se inspiraba en estas mismas ideas.

Ciertamente, para EE. UU. era un proyecto de guerra, aunque sea de guerra fría. Efectivamente, sobre la base de este proyecto EE. UU. ganó la guerra fría y derrotó el bloque soviético. Esta derrota se hizo obvia en el curso de los años 70 del siglo pasado, aunque todavía no se trataba del derrumbe.

Como era un proyecto de guerra en contra de la Unión Soviética, por supuesto no la incluyó, aunque haya sido su aliado durante la II Guerra Mundial. Se le otorgó un crédito de diez mil millones de dólares, pero ahora se exigió el pago de este crédito. Cuando la Unión Soviética lo rechazó, EE. UU. la denunció. No tomó siquiera en cuenta el hecho de que la Unión Soviética entera se movía al borde del hambre. 
George Soros, el gran especulador financiero, decía en una entrevista sobre este proyecto:

SPIEGEL ONLINE: ¿Qué esperaba usted de Europa, cuando la Segunda Guerra Mundial llegó a su fin? ¿Podía creer que el continente alguna vez encontraría paz?

SOROS: Nuestra gran suerte después del final de la guerra fue el Plan Marshall. Sin este plan Europa no sería pensable. Fue para ella una partera. El plan fue probablemente el proyecto de ayuda para el desarrollo más exitoso en la historia mundial, y mostró, qué tipo de buenos hechos podían hacer los Estados Unidos, que en ese entonces era ampliamente dominante como ahora lo es Alemania en Europa. Estados Unidos se concentró en la reconstrucción del continente, sobre el cual había conducido una guerra enconada. Esa es la gran diferencia con el comportamiento actual de Alemania: Estados Unidos estaba listo, tal vez no para olvidar los pecados del pasado, pero sí de perdonarlos. Alemania, por el contrario, parece que actúa sólo a través de sanciones y castigos, sin que el país ofrezca al resto de Europa una visión positiva similar a la que los estadounidenses ofrecieron también a los alemanes [Traducción propia] (Spiegel Online; 2014, 5 de marzo) ${ }^{3}$

El problema de toda esta política es que Soros no discute el hecho de que este proyecto muy eficiente, era un proyecto de guerra fría y no para la paz. Por eso ha tenido una cierta perversidad.

Si miramos esta estrategia de política económica de la posguerra, nos damos cuenta en seguida, que se trata de lo contrario de la estrategia de globalización introducida a partir de los años 80 del siglo pasado. Sin embargo, es la estrategia económica más exitosa que cualquier otro proyecto en el capitalismo occidental. Además logra combinar una estrategia muy dinámica de altas tasas de crecimiento con un desarrollo también único del Estado social, y por tanto, también de la democracia. Lo que más llama la atención es que ahora la política del Estado social es artificialmente debilitada y aparece por todos lados el intento de una política para eliminarlo lo más posible y sustituirlo por un Estado de seguridad, que tiene como centro el

\footnotetext{
${ }^{3}$ SPIEGEL ONLINE: Was erwarteten Sie eigentlich für Europa, als der Zweite Weltkrieg endlich zu Ende ging? Konnten Sie überhaupt daran glauben, dass der Kontinent jemals Frieden finden würde?. SOROS: Unser großes Glück nach dem Ende des Krieges war der Marshall-Plan. Ohne ihn ist die Europäische Union überhaupt nicht denkbar, er war ihr Geburtshelfer. Der Plan war wahrscheinlich das erfolgreichste Entwicklungshilfeprojekt der Weltgeschichte. Und er zeigte, zu welch guten Taten die Vereinigten Staaten fähig waren, die damals weltweit so dominant waren wie heute Deutschland in Europa. Amerika konzentrierte sich auf den Wiederaufbau des Kontinents, auf dem es gerade noch erbittert Krieg geführt hatte. Das ist der große Unterschied zum heutigen Verhalten Deutschlands: Amerika war bereit, die Sünden der Vergangenheit vielleicht nicht zu vergessen, aber zu vergeben. Deutschland hingegen scheint es bloß um Sanktionen und Strafe zu gehen, ohne dass das Land dem Rest Europas eine ähnlich positive Vision bietet, wie sie damals die Amerikaner auch den Deutschen boten. (Spiegel Online; 2014, 5 de marzo).
}

6

Franz Hinkelammert

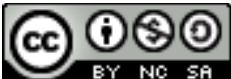

Revista Economía y Sociedad by Universidad Nacional is licensed under a Creative Commons Reconocimiento-NoComercialCompartirlgual 4.0 Internacional License. Creado a partir de la obra en http://www.revistas.una.ac.cr/index.php/economia 
aparato militar y los servicios secretos de seguridad. Es más notable hasta ahora en EE. UU. Pero toda esta política encuentra mucha resistencia de parte de la población, lo que explica que haya resultado mejor en los países con Dictaduras de Seguridad Nacional. Las sociedades europeas nunca después han logrado una dinámica ni comparable con este período de algo más de 30 años.

Sin embargo, la agresividad en contra del Estado social no ha disminuido a pesar de la resistencia popular. De hecho se trata de un gran ataque en contra de todo lo que podríamos llamar los derechos humanos de la vida humana, como habían surgido a partir de la revolución francesa. Son los grupos del poder económico quienes hacen el ataque, basándose en el hecho de que dominan prácticamente todos los medios de comunicación y los mecanismos de financiamiento de las elecciones e inclusive de determinados partidos políticos. Se trata de un gigantesco poder frente al cual no existe ningún control democrático. Es más bien el poder que controla prácticamente muchos de los mecanismos democráticos de control del poder.

Las posiciones, que en aquel tiempo se asumieron, son, si hoy se analizan, denunciados como izquierda extrema. Seguramente es extraño, si se piensa a Adenauer y Erhard como izquierda extrema, o a Pepe Figueres de Costa Rica.

De esta manera resulta un choque entre el mercado y el Estado social, pero a la vez con el necesario desarrollo de una intervención sistemática en los mercados en los dos lugares mencionados, el de los derechos humanos y de las necesidades ecológicas en cuanto a la naturaleza. Para entender mejor este choque, vamos a dar una interpretación breve de la revolución francesa, en la cual y a partir de la cual se mostró por primera vez en nuestra historia este choque.

\section{La estrategia de globalización y la meta de la eliminación de los derechos humanos de la vida humana}

Mencionamos anteriormente que todo el proyecto de reconstrucción de la Europa de posguerra era de hecho una medida de guerra en contra del socialismo soviético. En la década de los 70, sin embargo, era visible el hecho de que EE. UU. y sus aliados europeos habían ganado la guerra fría que habían promovido desde el final de la II Guerra Mundial.

Gastar dinero para promover una política de derechos humanos y el correspondiente desarrollo de un Estado social, aparentemente ya no valía la pena. El rostro humano del capitalismo había sido un arma de la guerra fría que ahora sobraba. Toda política de este tipo se declaraba rápidamente el enemigo de la libertad que había que derrotar. Eso empezó con el golpe militar chileno y su dictadura de Seguridad Nacional, con la llegada al gobierno de Margaret Thatcher en Inglaterra y posteriormente de Ronald Reagan en EE. UU. Se reducían ahora los impuestos 
sobre los ingresos altos, y se financiaba el déficit resultante por una disolución progresiva del Estado social. Las dictaduras de Seguridad Nacional tenían la estructura ideal para imponer cambios de este tipo, por medio de la pura represión policial. A esta represión policial se daba ahora el nombre de libertad; hasta el régimen de Pinochet era el régimen de una sociedad que por fin era libre. Sin embargo, en Inglaterra y EE. UU. se formaron mayorías de tendencia neoliberal a partir de los grupos de poder económico.

Todo eso se podía solamente hacer promoviendo una visión completamente contraria a la anterior, y se tenía el poder necesario sobre los medios. Como base ideológica sirvió, sin embargo, una teoría económica y una ideología correspondiente basada en el neoliberalismo como se había ya formado sobre todo en EE. UU. y allí en la escuela de Chicago.

El fundador de este pensamiento es Ludwig von Mises que dice con toda brutalidad de lo que se trata:

Se parte siempre de un error grave, pero muy extendido: el de que la naturaleza concedió a cada uno ciertos derechos inalienables, por el solo hecho de haber nacido. (Ludwig, 2011, pp.79-80)

De un golpe trata de borrar todo el desarrollo de los derechos humanos y toda emancipación humana posterior a la revolución francesa y todo desarrollo del Estado social para su promoción. Lo declara ahora para todo el mundo:

Si asiáticos y africanos no permitieron, por las razones que fuere, la aparición de un capitalismo autóctono allá ellos; ése es su problema. Occidente no tiene la culpa de nada; ya hizo bastante procurando, durante repetidas décadas, alumbrar la correcta vía (Ludwig, 2011. p.80).

Al mercado lo declara institución perfecta que no se debe intervenir o adaptar bajo ninguna condición. Es la utopía fatal del neoliberalismo y una de las utopías absolutamente destructoras. Solamente hay:

Para el alivio de tales males sólo una vía tienen franca: la implantación, sin reservas, del laissez faire capitalista. Lo que estos pueblos precisan es iniciativa privada y acumulación de nuevos capitales, o sea, ahorradores y empresarios (Ludwig, 2011, p. 80).

La ley del mercado es declarada ley absoluta y el mercado es ahora transformado en mercado total. A la vez toda acción humana, si es posible, tiene que ser privatizada, para que esté también penetrada por este laissez faire total. Lo que aparece es un Ilamado al totalitarismo del mercado.

8 
Es interesante el desarrollo de este argumento por Hayek durante su visita a Chile bajo la dictadura de Seguridad Nacional vigente allí en este tiempo:

Una sociedad libre requiere de ciertas morales que en última instancia se reducen a la mantención de vidas: no a la mantención de todas las vidas porque podría ser necesario sacrificar vidas individuales para preservar un número mayor de otras vidas. Por lo tanto las únicas reglas morales son las que llevan al 'cálculo de vidas': la propiedad y el contrato (Hayek, F.; 1981, 19 de abril)

Estos muertos los hace ver de la manera siguiente:

Al igual de los ancestros que habitaban cavernas, el hombre contemporáneo debe aceptar el control demográfico tradicional: hambrunas, pestes, mortalidad infantil, etcétera. (Hayek citado por Biagini, Fernández, 2015, p.172)

Pero este mismo pensamiento se introdujo masivamente en los movimientos socialdemócratas. Del candidato socialdemócrata de las últimas elecciones alemanes Peer Steinbrück para el parlamento se consta:

Peer Steinbrück (2015) enseña en su nuevo libro "Futuro postergado" a los socialdemócratas. Su consejo: Váyanse al centro. Con la elaboración del Estado de bienestar el partido hoy no está a la altura del tiempo.

Estado de bienestar aquí tiene el mismo significado que Estado social. El líder socialdemócrata abandona toda la tradición de derechos humanos, en la cual habían participado mucho precisamente los socialdemócratas de antes.

Además, para Steinbrück (2015) el extremismo del mercado total es el medio para pasar el centro. Si eso es así, ¿qué es entonces estar a la derecha del centro? Es el uso de lenguajes fraudulentos.

Lo que aquí se dice en nombre del neoliberalismo, lo encontramos ya en Primo de Rivera, el fundador de la Falange española: "Si escucho la palabra humanidad, tengo ganas a sacar la pistola". Cuando los fascistas ocuparon durante la guerra civil española en 1936 la Universidad de Salamanca, el general que dirigía la operación, gritaba: ¡Muera la inteligencia! ¡Viva la Muerte! En el neoliberalismo escuchamos lo mismo en otras palabras. En Heidegger el ser humano es un ser para la muerte. El neoliberalismo es el descendiente legítimo del pensamiento fascista. No es fascista, pero comparte la misma espiritualidad. 
Parecería que esta abolición de todo humanismo y de todos los derechos humanos de la vida humana ya es total. Sin embargo, eso no es cierto y por eso ahora aparece un conflicto muy violento. Se hacía visible en el actual conflicto en Inglaterra y en el partido Labour Party.

Con Corbyn aparece de repente un líder del partido laborista más bien de la izquierda, elegido por las bases del partido. Pero la burocracia del partido y sobre todo Blair se ponen violentamente en su contra. Con eso hacen un frente común con Cameron, el primer ministro conservador de Inglaterra.

Sin embargo, el conflicto de Cameron con Corbyn tuvo su lado sumamente interesante. Las perspectivas de las cuales habla Corbyn son perspectivas del reformismo de la posguerra. Pero en su primer pronunciamiento después de su elección hizo una declaración que llama la atención. Dice:

Nuestra tarea es demostrar, que la economía y nuestra sociedad pueden ser formadas de una manera tal, para que sirvan para todos. Eso significa, asegurar, que nos levantemos en contra de cualquier injusticia, donde la encontramos y de luchar por un futuro más honesto (fair) y más democrático, que satisface lo necesario para todos ${ }^{4}$. (Corbyn, 2015)

Lo que Corbyn desarrolla aquí, es la perspectiva que vale para todas las medidas que quiere tomar. Hace ver lo que Corbyn entiende como el sentido de toda su acción. Es interesante, que se base en dos fuentes. Cuando dice que "nos levantemos en contra de cualquier injusticia, donde la encontramos" cita a Marx con lo que Marx llama su imperativo categórico. Lo hace en su artículo "La introducción a la crítica de la filosofía del derecho de Hegel" del año 18445. La otra fuente aparece, cuando Corbyn dice: "luchar por un futuro más honesto (fair) y más democrático, que satisface lo necesario para todos". La fuente es, lo que dicen los zapatistas de México, es decir, se trata de "un mundo en el cual todos y todas tienen lugar" (y la naturaleza también).

Se nota que Corbyn expresa una espiritualidad.

Me parece eso muy esencial, porque para cualquier izquierda es esencial poner en el comienzo de su autointerpretación esta perspectiva de toda su acción y con eso el sentido de esta acción.

\footnotetext{
${ }^{4}$ Our job is to show that the economy and our society can be made to work for everyone. That means ensuring we stand up against injustice wherever we find it and we fight for a fairer and more democratic future that meets the needs of all. (Corbyn, 2015)

${ }^{5}$ Marx dice allí: "La crítica de la religión desemboca en la doctrina de que el hombre es el ser supremo (no: la esencia suprema) para el hombre y, por consiguiente, en el imperativo categórico de echar por tierra todas las relaciones en que el hombre sea un ser humillado, sojuzgado, abandonado y despreciable." (Marx, citado por Fromm, 1962, p. 230)
}

10

Franz Hinkelammert

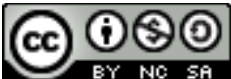

Revista Economía y Sociedad by Universidad Nacional is licensed under a Creative Commons Reconocimiento-NoComercial- 
Pero seguramente Corbyn tenía también presente los resultados sobre todo en América Latina de los gobiernos rebeldes que no aceptaron su sumisión a las ideologías neoliberales y que volvieron a una perspectiva de basar el desarrollo económico sobre el desarrollo ampliado del Estado social y sus políticas. Se trata de una experiencia de recuperación de un tipo de desarrollo que se había iniciado después de la II Guerra Mundial a partir de la reconstrucción de las economías de Europa occidental. Sin embargo, esta vez se trataba de un proyecto de paz, no de una guerra, aunque esta era una guerra fría. El capitalismo parece completamente incapaz para una acción de este tipo.

Se trata del proyecto de basar el desarrollo económico no en la privatización de todo, sino en la ampliación del Estado social. Con eso se recupera una manera de hacer la economía, que mostró ya en la posguerra su alta capacidad de desarrollo integral de los países. Esta política también ahora resulta altamente eficaz. La política del mercado total limita extraordinariamente la capacidad de los países de promover su desarrollo.

Hasta para China, que económicamente se ha desarrollado muy rápido, hoy aparecen los límites que probablemente ya no se pueden superar si no es basando por fin su desarrollo económico en un mucho mayor desarrollo del Estado social. Haber buscado su desarrollo en una política muy cercana al neoliberalismo va a significar un obstáculo para su desarrollo en el largo plazo. El mismo desarrollo económico se agota cuando no basa su estabilidad en la ampliación de un Estado social. La realidad económica confirma eso. A la larga no hay desarrollo económico sin desarrollo del Estado social y sus políticas. Además, la falta de hacer eso produce en gran parte la actual incapacidad de reaccionar ante el problema ecológico. Porque el Estado social es necesariamente un Estado que interviene constantemente en el mercado. También se necesita hoy este Estado intervencionista, si se quiere enfrentar la crisis ecológica. Actualmente, el neoliberalismo revela su carácter suicida.

La reacción en contra de Corbyn fue absolutamente condenatoria. Decía el ministro de defensa:

Ya sea debilitando nuestra defensa, subiendo impuestos, endeudándonos y gastando más o encareciendo el coste de vida a base de imprimir más dinero, el Partido Laborista de Jeremy Corbyn perjudicará a la gente trabajadora. (Fallon, citado por Guimón, 2015).

Pero Cameron dio el golpe más artero:

El Partido Laborista es ahora una amenaza a nuestra seguridad nacional, a nuestra seguridad económica y a la seguridad de vuestras familias. (Cameron, citado por Guimón, 2015) 
Usa el lenguaje de los años 80 del siglo pasado, cuando los gobiernos totalitarios de América Latina se llamaron dictaduras de Seguridad Nacional. Lo que dice de hecho, es que Corbyn es un peligro tan grande que frente al cual puede ser necesario levantar un nuevo Pinochet. Actúa en seguimiento de Margaret Thatcher. Cuando en el tiempo de su gobierno Pinochet estaba en prisión preventiva en Londres, ella lo visitó demostrativamente y lo llamó públicamente amigo de Gran Bretaña. En este contexto, hoy el programa político de Corbyn completamente legítimo es diabolizado por Cameron y amenazado. Cameron transforma el conflicto en un conflicto total y transforma la ideología del mercado en la ideología de un Mercado totalitario. Declara una guerra civil, aunque una guerra de tipo fría. Lo que comprueba precisamente es que un capitalismo neoliberal no se puede sostener a lo largo si no es como un sistema de mercado total sostenido por ideologías de Seguridad Nacional. No tiene argumentos válidos. Repite lo que estaba en su comienzo: el primer país que aplicó a fondo el programa neoliberal ha sido Chile cuando era dictadura de Seguridad Nacional bajo Pinochet. Si se quiere sostener, el neoliberalismo tiene que volver a basarse en tales dictaduras. Volver a las fuentes. El mismo Cameron se ha dado cuenta y lo fomenta. Amenaza a su adversario político con un nuevo Pinochet.

\section{La recuperación de los esfuerzos de emancipación humana}

Desde hace algún tiempo en América Latina está surgiendo un nuevo proyecto de sociedad alternativa, que responde directamente al totalitarismo del mercado que fomenta el neoliberalismo. El proyecto de Corbyn, que hemos citado antes, se inscribe obviamente en esta tradición. Su base es lo que presentaron en México los zapatistas como su perspectiva y que fue desarrollada después en toda América Latina: una sociedad en la que quepan todos y todas y la naturaleza también.

Esta es la visión alternativa en su forma objetiva. A la vez es elaborada en su forma subjetiva y aparece en diferentes formulaciones. Una es la que se elaboró en algunos países latinoamericanos a partir de una larga tradición indígena, como en Bolivia y Ecuador. En Bolivia se llama Suma Qamaña o Sumak Kawsay en Ecuador, lo que se puede traducir como buen vivir. No se trata del vivir bien aristotélico, que parte del individuo. Se trata más bien de una convivencia, sin la cual no se puede vivir bien. Es algo que en África del Sur se llama: yo soy, si tu eres, o yo soy si ustedes son o variaciones correspondientes. En África se llama el humanismo Ubuntu y tiene una larga tradición en muchas culturas africanas.

Las dos formulaciones coinciden. Una dice, lo que esta sociedad, de la cual se trata, es cuando la miramos desde afuera. La otra dice, lo que son los sujetos de esta sociedad, que son su soporte. Se trata de una exigencia y referencia de orientación en cada momento y en cada lugar. Es la perspectiva del ser humano como ser viviente. Es lo que está implícito al buen vivir boliviano. De hecho es la respuesta al proyecto neoliberal de una sociedad sin derechos humanos que tiene

12

Franz Hinkelammert

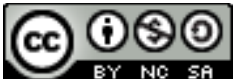

Revista Economía y Sociedad by Universidad Nacional is licensed under a Creative Commons Reconocimiento-NoComercial- 
solamente derechos del mercado, es decir, derechos que se pueden deducir del derecho de propiedad privada. Es la perspectiva del ser humano como capital humano, en relación al cual todos los que no sirven como capital humano o no quieren funcionar de esta manera, son considerados basura humana.

Hoy, este proyecto de un mundo en el cual quepan todos y todas entra en muchas discusiones. Entra también en las discusiones sobre la descolonización de la propia cultura. Como proyección puede precisamente definir lo que está ausente y hace falta en toda relación del ser humano con su ser cultural en todas sus relaciones con otros. Es decir, de lo que se trata como meta es del proceso de la descolonización de la propia cultura.

\section{Referencias}

Biagini, H; Fernändez, D. (2015). El neuroliberalismo y la ética del más fuerte. Recuperado de http://www.euna.una.ac.cr/index.php/100-categorias/lineas-editoriales/cienciassociales/251-el-neuroliberalismo-y-la-etica-del-mas-fuerte

Corbyn, J. (2015, September 13). Jeremy Corbyn: 'Britain can't cut its way to prosperity. We have to build it'. The Guardian. Recuperado de http://www.theguardian.com/commentisfree/2015/sep/13/jeremy-corbyn-labourleadership-victory-vision

Fromm, E. (1962). Marx y su concepto del hombre. Primera Edición en Español. Fondo de Cultura Económica. México. Recuperado de https://www.fondodeculturaeconomica.com/librerias/Detalle.aspx?ctit=014166E

Guimón, P. (2015, 14 de Septiembre). Cameron califica a Corbyn de "amenaza a la seguridad". El País. http://internacional.elpais.com/internacional/2015/09/13/actualidad/1442138786 $\underline{620188 . h t m l}$

Hayek, F. (1981, 19 de abril). "Entrevista". Justificando el genocidio de Pinochet en Chile. El Mercurio. Santiago, Chile.

Hinkelammert, F. (1981). Las armas ideológicas de la Muerte. Recuperado de http://www.pensamientocritico.info/index.php/libros/libros-de-franzhinkelammert/espanol 


\section{Economía}

Economía y Sociedad, Vol. 21, № 49, enero-junio 2016, pp. 1-14 EISSN: 2215-3403 URL http://www.revistas.una.ac.cr/economia

Ludwig, M. (2011). La mentalidad anticapitalista. Recuperado de http://unioneditorial.net/bibliotecaaustriaca?page $=$ shop. product details\&flypage=flypage.tpl\&product id=221\&catego ry id=6\&keyword=La+mentalidad+anticapitalista

Spiegel Online, (2014, 5 de marzo). Finanzinvestor und Merkel-Kritiker: Was George Soros antreibt. Zur Person. Recuperado de http://www.spiegel.de/wirtschaft/soziales/george-soros-mahnt-deutschengrossmut-gegenueber-krisenlaendern-an-a-956656.html

Steinbrück, P. (2015). Vertagte Zukunft: Die selbstzufriedene Republik. Recuperado de https://books.google.co.cr/books/about/Vertagte_Zukunft.html?id=c4jfrQEACAAJ\&r edir esc=y

14 